\title{
Efficacy and Safety of MLC601 in Patients with Mild to Moderate Alzheimer Disease: An Extension 4-Year Follow-Up Study
}

\author{
Hossein Pakdaman $^{a} \quad$ Koroush Gharagozli $^{a} \quad$ Mehdi Abbasi ${ }^{a}$ \\ Ali Sobhanian $^{a} \quad$ Ali Bakhshandehpour ${ }^{b}$ Farzad Ashrafi ${ }^{a} \quad$ Mitra Khalilzad $^{a}$ \\ Ali Amini Harandia \\ a Brain Mapping Research Center, Shahid Beheshti University of Medical Sciences, Theran, \\ Iran; ${ }^{b}$ Health and Human Physiology School, University of lowa, lowa City, IA, USA
}

\author{
Keywords \\ Alzheimer disease · MLC601 · Follow-up study · Safety
}

\begin{abstract}
Background and Aim: Alzheimer disease (AD) is the most common cause of dementia. Currently, there is no disease-modifying therapy for AD. We aimed to evaluate the long-term efficacy and safety of MLC601 in the treatment of AD. Methods: In this open-label extension study, patients with mild to moderate AD according to DSM-IV criteria were recruited. Patients received MLC601 capsules 3 times a day for 4 years. Cognitive function was assessed every 6 months using Mini-Mental State Examination (MMSE) and Alzheimer's Disease Assessment Scale-Cognitive Subscale (ADAS-Cog) scores. Safety profiles, including adverse events (AEs), and treatment-related abnormality in laboratory tests were also reported. Results: Of a total of 122 patients, 105 completed the study. The mean age was $66.8 \pm 6.3$ years at the beginning of the study. Sixty-five (61.9\%) were female. The mean ( \pm SD) change in MMSE and ADAS-Cog scores at the end of the study was $2.1( \pm 3.8)$ and $-5.1( \pm 8.7)$, respectively. Repeated measure analysis revealed a statistically significant change in both scores $(p<0.001)$. No patient left the study due to an AE. No abnormality was noted in lab tests. Gastrointestinal symptoms were the most commonly reported AEs. Conclusion: The efficacy of treating AD patients with MLC601 over 4 years has been demonstrated in the present study. Overall, it seems that the safety and efficacy of MLC601 is promising compared to currently prescribed treatments.
\end{abstract}


Pakdaman et al.: Efficacy and Safety of MLC601 in Patients with Mild to Moderate Alzheimer Disease: An Extension 4-Year Follow-Up Study

\section{Introduction}

Alzheimer disease (AD) is the most common cause of dementia, accounting for almost $70 \%$ of the cases. The prevalence of AD also continues to rise. Thus, it has been estimated that due to increased life expectancy the number of patients with memory loss will reach 131.5 million worldwide by the end of $2050[1,2]$. While our knowledge regarding the pathogenesis of AD has increased during the past decade, our treatment options are still confined to only a few drugs. Currently, acetylcholinesterase inhibitors (AChEIs) are widely used and accepted as a treatment for AD. AChEIs have been used to slow the progression of cognitive decline in $\mathrm{AD}$, and some improvement has been reported on cognition scores [3], but they cannot cure AD. Some believe that they only provide symptomatic relief for a short period without reducing or halting pathological hallmarks of $\mathrm{AD}$, including formation of neurofibrillary tangles and amyloid plaques. Also, AChEIs are well known for inducing cholinergic side effects [4-6], which could be severe as confirmed in a recently published drug surveillance survey analyzing national databases in the US and Canada [7]. Consequently, new treatments which can prevent or delay the onset of $\mathrm{AD}$, or which can slow the progression of this disorder, are needed [8]. MLC601 (NeuroAiD ${ }^{\mathrm{TM}}$, Moleac Pte. Ltd., Singapore) is a natural medicine which contains both herbal and animal products, and it has been reported to have both neuroprotective and neuroregenerative properties $[9,10]$. The present study was carried out to evaluate the long-term efficacy and safety of MLC601 in patients with mild to moderate AD. The rationale behind this study is supported by current evidence on the long-term efficacy of MLC601 in poststroke recovery, by positive results in the treatment of dementia and minimal cognitive impairment [11-14], and by the possible role of MLC601 in the modulation of the amyloid precursor protein and in decreasing tau phosphorylation $[15,16]$. Therefore, we speculate that treatment with MLC601 could be beneficial to patients at early stages of AD.

\section{Methods}

This was an open-label extension study evaluating the efficacy and safety of MLC601 in patients with mild to moderate AD. Inclusion and exclusion criteria have previously been described [13]. Inclusion criteria were age above 50 years, a diagnosis of AD according to DSM-IV criteria, mild to moderate AD with Mini-Mental State Examination (MMSE) scores ranging from 10 to 24, and nonresponders to rivastigmine. Exclusion criteria were as follows: uncontrolled diabetes mellitus, hypertension, unstable cardiac disease, severe obstructive pulmonary disease, renal or hepatic failure, and/or other life-threatening conditions, as well as any other causes of cognitive impairment. After completion of our previous study [13] in which subjects had been treated with MLC601 for 18 months, recruited patients were prospectively followed to assess the efficacy and safety of MLC601 in the long term. Patients received MLC601 capsules (NeuroAiD ${ }^{\mathrm{TM}}$; 0.4 g per capsule) 3 times a day. Efficacy was determined by evaluating cognitive function using the Alzheimer's Disease Assessment Scale-Cognitive Subscale (ADAS-Cog) and the MMSE. Assessments took place every 6 months up to 4 years. Patients who failed to come for any of the follow-up visits were excluded from the study. Adverse events were also recorded at each follow-up visit. Furthermore, abnormal findings in physical examinations or lab tests, including complete blood count, liver function tests, and renal function tests, were documented. Neurologists performed evaluations as well.

The MMSE is widely used to evaluate cognitive function. It examines multiple domains, and its score ranges from 0 (severe impairment) to 30 (no impairment) [17]. The ADAS-Cog is also one of the most popular measures for AD. It contains 11 tasks, and its score ranges from 0 to 70 with higher scores indicating increased severity of the condition [18]. MLC601 is a 
Table 1. MMSE and ADAS-Cog scores in patients treated with MLC601

\begin{tabular}{lrrrrrrrrrr}
\hline & Baseline & 6 months & 12 months & 18 months & 24 months & 30 months & 36 months & 42 months & 48 months & $p$ value \\
\hline MMSE & $18.9 \pm 5.4$ & $18.4 \pm 4.7$ & $19.2 \pm 5.0$ & $19.1 \pm 5.3$ & $18.9 \pm 5.2$ & $18.3 \pm 5.5$ & $17.2 \pm 5.4$ & $16.8 \pm 5.1$ & $16.3 \pm 4.8$ & $<0.001$ \\
ADAS-Cog & $21.5 \pm 8.4$ & $22.6 \pm 8.2$ & $20 \pm 8.3$ & $20.8 \pm 8.5$ & $21.5 \pm 8.4$ & $22.5 \pm 8.8$ & $24.6 \pm 9.3$ & $25.3 \pm 10.3$ & $27.7 \pm 12.8$ & $<0.001$ \\
\hline
\end{tabular}

Values are means \pm standard deviations. MMSE, Mini-Mental State Examination; ADAS-Cog, Alzheimer's Disease Assessment Scale-Cognitive Subscale.

Table 2. Adverse events in patients treated with MLC601

\begin{tabular}{lc}
\hline & $\begin{array}{l}\text { Patients, } n(\%) \\
(n=122)\end{array}$ \\
\hline Nausea & $3(2.5)$ \\
Anorexia & $2(1.6)$ \\
Bloating & $5(4.1)$ \\
Abdominal cramps & $4(3.3)$ \\
Abdominal pain & $14(11.5)$ \\
Heartburn & $10(8.2)$ \\
Fatigue & $4(3.3)$ \\
Hypersalivation & $1(0.8)$ \\
Constipation & $2(1.6)$ \\
Lightheadedness & $2(1.6)$ \\
Headache & $5(4.1)$ \\
\hline
\end{tabular}

traditional Chinese medicine, and it is composed of both animal $(0.0665 \mathrm{~g}$ Hirudo, $0.0285 \mathrm{~g}$ Cornu saigae tataricae, $0.095 \mathrm{~g}$ Mesobuthus martensii, $0.0665 \mathrm{~g}$ Eupolyphaga seu steleophaga, and $0.0285 \mathrm{~g}$ Calculus bovis artifactus) and herbal (0.114 g radix Paeoniae rubrae, $0.57 \mathrm{~g}$ radix Astragali, 0.114 g radix Salviae miltiorrhizae, 0.114 g rhizoma Chuanxiong, 0.114 g radix Angelicae sinensis, 0.114 g radix Polygalae, 0.114 g Prunus persica, 0.114 g Carthamus tinctorius, and $0.114 \mathrm{~g}$ rhizoma Acori tatarinowii) products [9].

The study protocol was approved by the Ethics Committee of Shahid Beheshti University of Medical Sciences. It was also in accordance with the Helsinki Declaration and its later amendments. Patients or their caregivers gave their informed consent before participating in this study, and patients were allowed to leave the study without any concern regarding receiving treatment from their physicians. Data analysis was performed using SPSS 18.0, and descriptive data were reported as means \pm standard deviations. When appropriate, repeated measures analysis was conducted to interpret data. A $p$ value $<0.05$ was considered statistically significant.

\section{Results}

Of a total of 122 patients, 105 completed the study. Nine patients left the study due to personal reasons, and 8 patients dropped out due to cerebrovascular accident ( 3 patients), myocardial infarction ( 2 patients), pneumonia ( 2 patient), and a car accident ( 1 patient). The mean age was $66.8 \pm 6.3$ years at the beginning of the study. Sixty-five patients (61.9\%) were female, and 40 patients (38.1\%) were male. The mean scores for both the MMSE and ADAS-Cog remained almost the same during the first 5 visits and then changed gradually towards a worsening in disease severity (Table 1).

The mean $( \pm \mathrm{SD})$ change in MMSE and ADAS-Cog scores at the end of the study was 2.1 $( \pm 3.8)$ and $-5.1( \pm 8.7)$, respectively. Repeated measure analysis revealed a statistically signif- 
Pakdaman et al.: Efficacy and Safety of MLC601 in Patients with Mild to Moderate Alzheimer Disease: An Extension 4-Year Follow-Up Study

icant change in both scores with ADAS-Cog scores showing greater change than the MMSE scores $(p<0.001)$.

Adverse events were reported in 18 patients, and they were mild in severity. No patients discontinued therapy due to adverse events related to the study treatment according to the investigators' opinion. Gastrointestinal symptoms were the most commonly reported adverse events. Table 2 summarizes adverse events in the study group. Additionally, no abnormalities were noted in lab tests.

\section{Discussion}

Limited data are available on the efficacy and safety of MLC601 in the treatment of patients with AD. This is the first study evaluating the long-term benefits of MLC601 in patients with mild to moderate AD. Our results show that MLC601 is a well-tolerated therapy with mild and temporary adverse events. However, the efficacy of the therapy tends to decline over time. It is worth noting that during the first 2.5 years of the treatment, patients experienced almost no worsening of their cognitive function. Combining the results of the current report and of our previous study with the same group of patients demonstrates the promising effect of MLC601 in slowing the progression of AD to more severe types. Therefore, patients with a mild to moderate form of AD would benefit from an early treatment with MLC601 which is more tolerable compared to conventional AChEIs.

To the date of this article's publication, no study has evaluated the long-term efficacy and safety of MLC601 in patients with AD. Therefore, a comparison of the findings of this report to other studies is not possible. Also, in the current literature, only a few studies have reported the efficacy of AChEIs, as an approved treatment for AD, in the long term. In an open-label phase 3 extension study by Doody et al. [19], 763 patients with mild to moderate AD were treated with donepezil. After 3 years of treatment, the cumulative mean change in ADAS-Cog scores from baseline was around 10 in the donepezil $5 \mathrm{mg} /$ day group and 12 in the donepezil $10 \mathrm{mg} /$ day group. However, the number of patients who discontinued treatment was low (128 patients). Comparing our findings to the results of that study, MLC601 showed a better outcome (2.7 points change in the mean ADAS-Cog scores) in the same time period. The authors also reported transient and tolerable adverse events with no need for adjustment of the treatment dosage. Adverse events happened in $95 \%$ of the patients, and they were mostly related to the nervous system and the digestive system. Urinary tract infection, accidents, pain, agitation, and diarrhea were the most frequently reported adverse events [19]. In comparison, the safety profile of MLC601 in our study is superior to donepezil. This is because a much lower number of patients reported adverse events in our study, and the prevalence of the nervous system-related symptoms was considerably lower.

In another study, 37 patients with mild to moderate AD received rivastigmine for up to 234 weeks [20]. Two-thirds of the patients were still enrolled in the study at the end of the follow-up period. The authors reported that the mean ADAS-Cog scores during the study were 24.66, and the mean change in ADAS-Cog scores from the baseline was around 8 points (interpreted from the presented figure in the article). Seven patients left the study due to adverse events, and 1 patient cited treatment failure as the reason for termination. The authors conclude that rivastigmine is a well-tolerated therapy with long-term efficacy in AD patients. However, compared to our findings in the present study, their results are inferior both in terms of effectiveness and safety. Overall, the mean change in ADAS-Cog scores, in our study, was 5.1 versus 8 in their study. Also, no patient left our study due to adverse events related to study treatment [20]. 
Pakdaman et al.: Efficacy and Safety of MLC601 in Patients with Mild to Moderate

The encouraging efficacy of MLC601 is probably due to its role in the modulation of the amyloid precursor protein. It is also worth mentioning that MLC601 has been suggested as an alternative option for the treatment of $\mathrm{AD}$ and poststroke dementia [15]. Animal studies have also shown that MLC901, the herbal-only version of MLC601, has neuroprotective and neurorestorative properties. MLC901 decreases cognitive impairment following traumatic brain injury, and with its proneurogeneic effects, it increases the number of mature neuron cells in the hippocampus [21,22]. MLC901 also decreases tau phosphorylation at epitopes, which is associated with neurofibrillary tangle formation in AD [16]. In our previous study, we compared MLC601 with 3 conventionally prescribed AChEIs, including donepezil, rivastigmine, and galantamine. Over 16 months of the study, the mean change in MMSE and ADAS-Cog scores was not different between the treatment groups $(p=0.92$ for MMSE and $p=0.87$ for ADAS-Cog). The present study demonstrated that in a short follow-up period, the efficacy of MLC601 in patients with mild to moderate AD is equal to AChEIs [12]. The safety profile of MLC601 in our study confirms the finding of previous studies which reported transient, tolerable adverse events of MLC601. It is also worth pointing out that in the present study, as in previous reports, gastrointestinal symptoms were the most common adverse events of MLC601 [9, 12, 13, 23, 24].

There are several limitations as well as several strengths to our study. Having a relatively large population, a long duration of the follow-up, and detailed inclusion criteria are obvious strengths of this report. Limitations of the current study include lack of a placebo group, which could lead to an overestimation or underestimation of the efficacy of MLC601. Also, a heterogeneous pattern of disease severity in the present study makes detecting the efficacy of MLC601 challenging and drawing reliable conclusions difficult. In open-label studies, patients with good responses may continue their treatment, and the results of these studies may not represent whether the treatment is effective or not. Furthermore, only cognitive outcomes were evaluated in our study, so cognitive benefits of MLC601 cannot be extended to noncognitive domains. It is also worth mentioning that the cognitive batteries utilized in the present study have also been shown to have drawbacks in patients with AD. Therefore, randomized, placebo-controlled trials based on disease severity with a larger sample size, different cognitive batteries, and shorter intervals of follow-up visits are required to replicate the results of the pesent study.

\section{Conclusion}

The benefits of treating patients with mild to moderate AD with MLC601 over 4 years have been demonstrated in the present study. Overall, the efficacy of MLC601 is comparable to the efficacy of AChEIs, while it is better tolerated. Also, further studies on this population using a randomized controlled trial design are needed to validate the outcomes of this study.

\section{Acknowledgement}

The authors would like to thank the Clinical Research Development Unit (CRDU) of Loghman Hakim Hospital, Shahid Beheshti University of Medical Sciences, Tehran, Iran, for their support, cooperation, and assistance throughout the period of the study.

\section{Disclosure Statement}

All authors declare that they have no conflicts of interest to report. 
Pakdaman et al.: Efficacy and Safety of MLC601 in Patients with Mild to Moderate Alzheimer Disease: An Extension 4-Year Follow-Up Study

\section{References}

1 Prince M, Comas-Herrera A, Knapp M, Guerchet M, Karagiannidou M: World Alzheimer Report 2016: Improving Healthcare for People Living with Dementia: Coverage, Quality and Costs Now and in the Future. London, Alzheimer's Disease International (ADI), 2016.

-2 Brookmeyer R, Evans DA, Hebert L, Langa KM, Heeringa SG, Plassman BL, Kukull WA: National estimates of the prevalence of Alzheimer's disease in the United States. Alzheimers Dement 2011;7:61-73.

-3 Mohammad D, Chan P, Bradley J, Lanctôt K, Herrmann N: Acetylcholinesterase inhibitors for treating dementia symptoms - a safety evaluation. Expert Opin Drug Saf 2017;16:1009-1019.

4 Ferreira-Vieira TH, Guimaraes IM, Silva FR, Ribeiro FM: Alzheimer's disease: targeting the cholinergic system. Curr Neuropharmacol 2016;14:101-115.

5 Van der Schyf CJ: The use of multi-target drugs in the treatment of neurodegenerative diseases. Expert Rev Clin Pharmacol 2011;4:293-298.

6 Schmidt R, Hofer E, Bouwman F, Buerger K, Cordonnier C, Fladby T, Galimberti D, Georges J, Heneka M, Hort J: EFNS-ENS/EAN guideline on concomitant use of cholinesterase inhibitors and memantine in moderate to severe Alzheimer's disease. Eur J Neurol 2015;22:889-898.

-7 Ali TB, Schleret TR, Reilly BM, Chen WY, Abagyan R: Adverse effects of cholinesterase inhibitors in dementia, according to the pharmacovigilance databases of the United-States and Canada. PLoS One 2015;10:e144337.

-8 Scheltens P, Blennow K, Breteler MMB, de Strooper B, Frisoni GB, Salloway S, Van der Flier WM: Alzheimer's disease. Lancet 2016;388:505-517.

-9 Harandi A, Abolfazli R, Hatemian A, Ghragozlee K, Ghaffar-Pour M, Karimi M, Shahbegi S, Pakdaman H, Tabasi M, Tabatabae AL, Nourian A: Safety and efficacy of MLC601 in Iranian patients after stroke: a double-blind, placebo-controlled clinical trial. Stroke Res Treat 2011;2011:721613.

10 Heurteaux C, Gandin C, Borsotto M, Widmann C, Brau F, Lhuillier M, Onteniente B, Lazdunski M: Neuroprotective and neuroproliferative activities of NeuroAid (MLC601, MLC901), a Chinese medicine, in vitro and in vivo. Neuropharmacology 2010;58:987-1001.

-11 Venketasubramanian N, Young SH, Tay SS, Umapathi T, Lao AY, Gan HH, Baroque I, Navarro JC, Chang HM, Advincula JM: Chinese medicine NeuroAiD efficacy on stroke recovery-extension study (CHIMES-E): a multicenter study of long-term efficacy. Cerebrovasc Dis 2015;39:309-318.

$\checkmark 12$ Pakdaman H, Harandi AA, Hatamian H, Tabatabae M, Delavar Kasmaei H, Ghassemi A, Gharagozli K, Ashrafi F, Emami Naeini P, Tavakolian M: Effectiveness and safety of MLC601 in the treatment of mild to moderate Alzheimer's disease: a multicenter, randomized controlled trial. Dement Geriatr Cogn Disord Extra 2015;5: 96-106.

13 Harandi AA, Ashrafi F, Tabatabaei M, Ghorbani A, Hatamian H, Pakdaman H, Ilkhani M, Gharagozli K, Shojae M, Asadollahi M: Efficacy and tolerability of MLC601 in patients with mild to moderate Alzheimer disease who were unable to tolerate or failed to benefit from treatment with rivastigmine. Br J Med Res 2013;3:341-350.

14 Pakdaman H, Harandi AA, Abbasi M, Kasmaei HD, Ashrafi F, Gharagozli K, Assarzadegan F, Behnam B, Arabahmadi M: Efficacy and safety of MLC601 in the treatment of mild cognitive impairment: a pilot, randomized, double-blind, placebo-controlled study. Dement Geriatr Cogn Dis Extra 2017;7:136-142.

15 Lim Y, Murray L, Lai M, Chen C: NeuroAiD ${ }^{\circledR}$ (MLC601) and amyloid precursor protein processing. Cerebrovasc Dis 2013;35:30-37.

16 Lee WT, Hsian CCL, Lim Y-A: The effects of MLC901 on tau phosphorylation. Neuroreport 2017;28:10431048.

17 Folstein MF, Folstein SE, McHugh PR: "Mini-mental state”: a practical method for grading the cognitive state of patients for the clinician. J Psychiatr Res 1975;12:189-198.

18 Weyer G, Erzigkeit H, Kanowski S, Ihl R, Hadler D: Alzheimer's Disease Assessment Scale: reliability and validity in a multicenter clinical trial. Int Psychogeriatr 1997;9:123-138.

19 Doody RS, Geldmacher DS, Gordon B, Perdomo CA, Pratt RD: Open-label, multicenter, phase 3 extension study of the safety and efficacy of donepezil in patients with Alzheimer disease. Arch Neurol 2001;58:427-433.

20 Farlow MR, Lilly ML: Rivastigmine: an open-label, observational study of safety and effectiveness in treating patients with Alzheimer's disease for up to 5 years. BMC Geriatr 2005;5:3.

21 Quintard H, Lorivel T, Gandin C, Lazdunski M, Heurteaux C: MLC901, a traditional Chinese medicine induces neuroprotective and neuroregenerative benefits after traumatic brain injury in rats. Neuroscience 2014;277: 72-86.

22 Lorivel T, Gandin C, Veyssière J, Lazdunski M, Heurteaux C: Positive effects of the traditional Chinese medicine MLC901 in cognitive tasks. J Neurosci Res 2015;93:1648-1663.

23 Siddiqui FJ, Venketasubramanian N, Chan E-Y, Chen C: Efficacy and safety of MLC601 (NeuroAiD ${ }^{\circledR}$ ), a traditional Chinese medicine, in poststroke recovery: a systematic review. Cerebrovasc Dis 2013;35:8-17.

24 Pakdaman H, Harandi AA, Gharagozli K, Abbasi M, Ghaffarpour M, Ashrafi F, Kasmaei HD, Harandi AA: MLC601 in vascular dementia: an efficacy and safety pilot study. Neuropsychiatr Dis Treat 2017;13:2551. 